\title{
A RARE CASE OF HETEROTOPIC PREGNANCY FOLLOWING OVULATION INDUCTION
}

Parinita Khot ${ }^{1}$, Nitin Kshirsagar², Digvijay Kadam³ ${ }^{3}$, Richa Priyamvada ${ }^{4}$

HOW TO CITE THIS ARTICLE:

Parinita Khot, Nitin Kshirsagar, Digvijay Kadam, Richa Priyamvada. "A Rare Case of Heterotopic Pregnancy following Ovulation Induction". Journal of Evolution of Medical and Dental Sciences 2015; Vol. 4, Issue 86, October 26; Page: 15100-15104, DOI: 10.14260/jemds/2015/2144

ABSTRACT: Heterotopic pregnancy is defined as the simultaneous gestation at two or more implantation sites. It is a rare clinical condition and can be life threatening due to the risk of rupture of the ectopic pregnancy. Clinicians and sonographers tend to be lulled into a false sense of security when an intrauterine gestation sac is seen. This results in inadequate inspection of the adnexa and remaining structures during ultrasonography. Every gynaecologist treating a woman of reproductive age group should consider the suspicion of heterotopic pregnancy. Even in the absence of any known risk factor, it can lead to significant morbidity and mortality. Prompt diagnosis and treatment will help in continuing the intrauterine pregnancy till term. We report a case of a 36 year third gravida who presented with heterotopic pregnancy on ultrasonography after taking clomifene citrate for two cycles. Patient was posted for laparotomy and unilateral salpingectomy was performed.

KEYWORDS: Heterotopic pregnancy, ectopic pregnancy, ovulation induction.

INTRODUCTION: Heterotopic pregnancy (HP) is the existence of 2 (or more) simultaneous pregnancies with separate implantation sites, one of which is ectopic. The estimated incidence in the general population is estimated at 1:30,000 (for a naturally conceived pregnancy).[1] With increasing usage of assisted reproduction, the frequency of heterotopic pregnancies was cited to be between 1:100 and 1:7,000.[1,2] For patients who have been treated with ovulation-induction medication an incidence of $33 / 10,000$ has been reported. ${ }^{[3]}$ while it is extremely rare among women who conceive naturally. This raised frequency has been attributed to several factors including higher incidence of pelvic inflammatory disease (PID) and the extended use of assisted reproductive technologies (ARTs). Heterotopic pregnancies have been diagnosed from 5-34 weeks of gestation with highest incidence at 5 to 8 weeks $(70 \%)$ and only $10 \%$ after the 11 th week. ${ }^{[4]}$ The mainstay of treating heterotopic pregnancy remains surgery while laparoscopy is also used these days.

CASE REPORT: A 36 years third gravida (P1, L1, A1) presented to the obstetric ward at Krishna hospital Karad with amenorrhoea of one and half months and an early ultrasonography showing a single live intrauterine gestation of 6 weeks and 5 days. It also showed a mixed echogenic mass lesion in right adnexal region measuring $6.4 \times 3.0 \times 3.5 \mathrm{~cm}$.

There was evidence of irregular solid component at periphery with echogenic tissue around showing ring vascularity. There is evidence of moderate fluid collection in the pouch of Doughlas and flanks. The patient had a previous vaginal delivery 7years back and was on ovulation induction drugs (Clomifene citrate) since two months for secondary infertility. Patient had been previously operated for left ovarian cyst 3 years back, details of which were not available.

On examination, patient was haemodynamically stable with a pulse of $88 / \mathrm{min}$ and B.P 120/80 $\mathrm{mmHg}$. She was mildly pale with haemoglobin of $9 \mathrm{gm} \%$. Abdomen was soft and showed no signs of tenderness. There were no specific findings on per speculum and per vaginal examination. 


\section{CASE REPORT}

As patient was anxious to continue the intrauterine pregnancy, patient was posted for laparotomy under general anaesthesia.

Intraoperative findings confirmed a right ruptured tubal ectopic pregnancy with haemoperitoneum of about $200 \mathrm{ml}$ with blood clots of around $50 \mathrm{gm}$. Left ovary showed the presence of corpus luteum.

The uterus corresponded to the period of gestation. Right sided salpingectomy was performed. Care was taken to prevent any manipulation of the uterus. Paracetamol was used for postoperative pain. The material was sent for definitive histologic examination and was confirmed chorionic villi suggestive of a tubal EP. The postoperative course was uneventful.

During the follow up on the second postoperative day, the ultrasound was repeated and a viable intrauterine pregnancy was seen, confirmed by the foetal cardiac activity. The patient was discharged on the 7 th postoperative day.
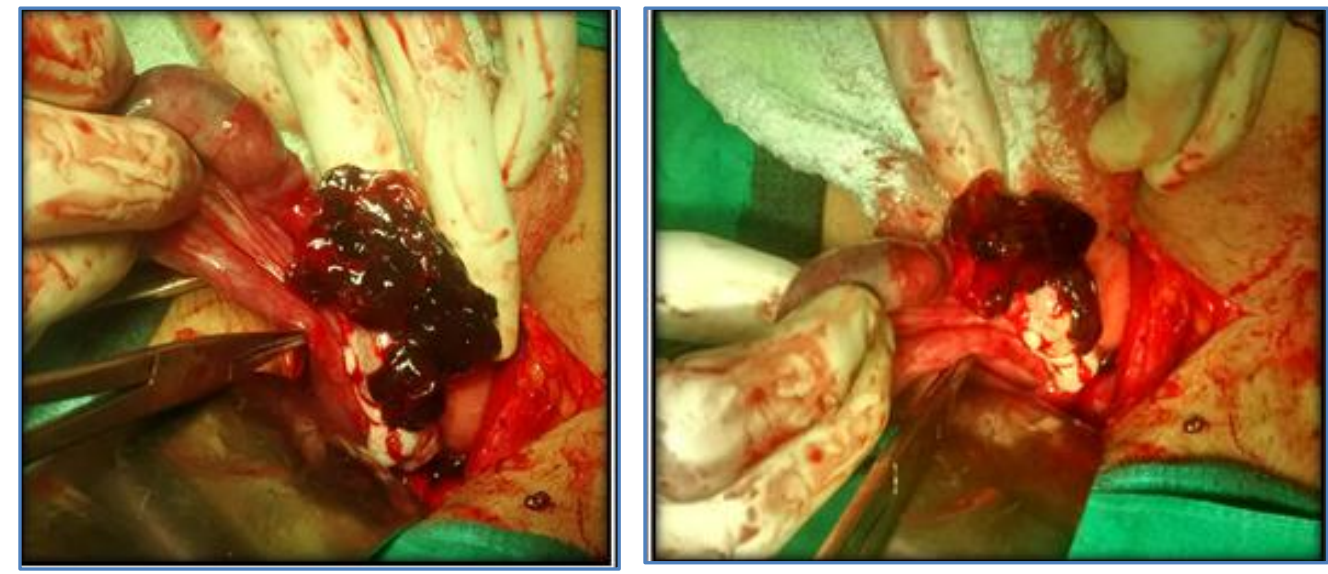

TUBAL ECTOPIC PREGNANCY THAT WAS RUPTURED

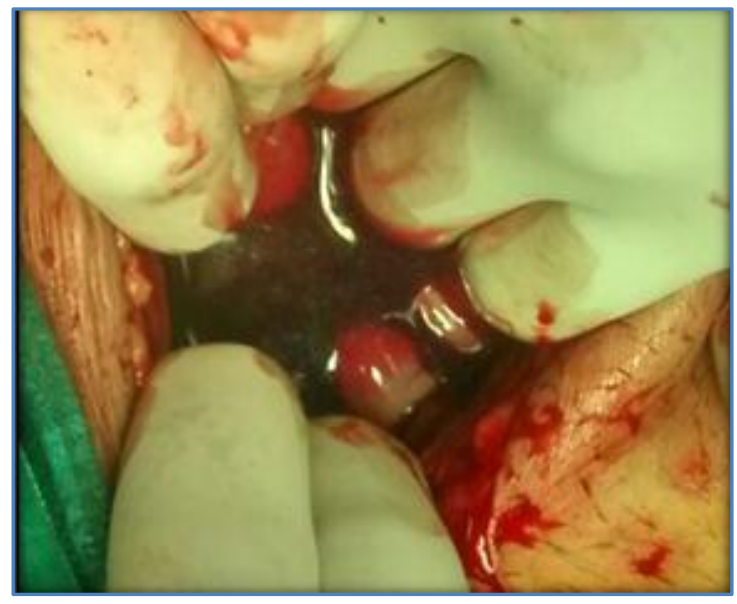

INTRAOPERATIVE HAEMOPERITONEUM

DISCUSSION: Heterotopic pregnancies presents as an intrauterine pregnancy and tubal, abdominal, corneal, cervical or ovarian pregnancy. A previous review showed that most of extra uterine pregnancies were located in the fallopian tube (72.5\%).[5] Risk factors. 


\section{CASE REPORT}

\section{Recognised Risk Factors predisposing to this condition include:}

- Use of an intrauterine contraceptive device.

- Prior tubal surgery.

- History of pelvic inflammatory disease.

- History of a previous ectopic pregnancy.

- Use of ovulation induction drugs/assisted reproductive techniques.

Spontaneous HP is a potential fatal condition. Clinicians should maintain a high index of suspicion in all patients presenting with amenorrhea, abdominal pain, adnexal mass, peritoneal irritation, and enlarged uterus, even if an IUP has been confirmed.[6]

A good history is important to identify risk factors for heterotopic pregnancy such as fertility treatment and tubal pathologies like pelvic inflammatory disease, endometriosis or previous tubal surgeries.[1]

Vaginal bleeding occurs rarely in heterotopic pregnancy, compared to ectopic pregnancy.[7] Pain and bleeding may be misdiagnosed as threatened abortion and thus cause a delay in the diagnosis of HP. The presence of an IUP, either viable or not, may actually mask the ectopic component of an HP.

\section{The differential diagnosis of abdominal pain in early pregnancy include:}

- Ruptured Ectopic pregnancy.[8]

- Ovarian torsion and ruptured corpus luteum cyst.

- -ub chorionic haemorrhage, caused by a partial detachment of the trophoblast from the uterus.[9]

- Acute appendicitis.

- Spontaneous abortion.

Health care professionals have been indoctrinated with the famous dictum by the French surgeon Henri Mondor (1885 - 1962) 'Think Ectopic' when assessing acute abdomen of pregnant women. Heterotopic pregnancies can pose a diagnostic dilemma because an early transvaginal ultrasound may not diagnose an ectopic gestation in all cases. Sometimes the presence of a haemorrhagic corpus luteum can confuse and delay the diagnosis of a heterotopic pregnancy.[10]

The ultrasound visualization of heart activity in both intrauterine and extra uterine gestations is important for diagnosis, but rare. In patients treated with ART, ovarian hyper stimulation syndrome is a frequent occurrence that can mask the presence of an EP.[5]

The detection rate of heterotopic pregnancy can vary from 41 to $84 \%$ with transvaginal ultrasound scans.[11,12] Also a high index of suspicion is necessary in the low risk symptomatic patient with abdominal or pelvic pain in which ultrasound findings are consistent with intrauterine gestation sac while free fluid is noted in the pelvis with or without an adnexal mass.

The diagnostic role of serum beta HCG levels in heterotopic pregnancy is debatable.[13] The combined use of serum beta human chorionic gonadotropin(HCG) level along with transvaginal ultrasound improved the sensitivity to diagnose intrauterine pregnancy to $100 \% .{ }^{[14]}$ the intrauterine pregnancy masks any underlying $\beta$-hCG changes from the extra uterine pregnancy and vice versa.

Medical management, although used successfully for an ectopic pregnancy, has a limited role in the management of heterotopic pregnancy. In cases similar to our case with ruptured ectopic pregnancy, the standard treatment is surgical removal of the ectopic either by laparoscopy or laparotomy with minimal manipulation of the uterus.[15] The successful non-surgical management of six cases of HTP using potassium chloride $(\mathrm{KCl})$ injection into the tubal EP has been reported.[16] 
CONCLUSION: The incidence of heterotopic pregnancies increased in recent years because of the escalating use of new reproductive technologies in infertile patients.[17] A HP following CC stimulation is an unusual phenomenon that can be associated with high morbidity and adverse consequences for future fertility.

The diagnosis may be challenging but may be determined by combining a pertinent history of risk factors, clinical symptoms, $\beta$-HCG titres and ultra-sonographic findings when indicative of free fluid in the pouch of Doughlas.

The diagnosis is confirmed at time of surgery and by histopathologic study. In patients undergoing fertility treatment, a high index of suspicion is the mainstay even in the presence of an intrauterine pregnancy and in asymptomatic patients.

ACKNOWLEDGEMENT: We thank Medical director KIMSDU, KARAD and head of department of OBGYN, for allowing us to use and publish the data related to the patient.

\section{REFERENCES:}

1. Mj G, R R. Heterotopic pregnancy in natural conception. J Hum Reprod Sci. 2008; 1(1): 37-8.

2. Press GM, Martinez A. Heterotopic pregnancy diagnosed by emergency ultrasound. J Emerg Med. 2007; 33(1):25-27.

3. J. L. Yovich, S. C. McColm, S. R. Turner, and P. L. Matson, "Heterotopic pregnancy from in vitro fertilization," Journal of In Vitro Fertilization and Embryo Transfer, vol. 2, no. 3, pp. 146-150, 1985.

4. Hassani KI, Bouazzaoui AE, Khatouf M et-al. Heterotopic pregnancy: A diagnosis we should suspect more often. J Emerg Trauma Shock. 2010; 3(3): 304. doi:10.4103/0974-2700.66563.

5. G. Barrenetxea, L. Barinaga-Rementeria, D. E. Lopez, J. A. Agirregoikoa, M. Mandiola, and K. Carbonero, "Heterotopic pregnancy: two cases and a comparative review," Fertility and Sterility, vol. 87, no. 2, pp. 417.e9-417.e15, 2007.

6. Varras M, Akrivis C, Hadjopoulos G, Antoniou N. Heterotopic pregnancy in a natural conception cycle presenting with tubal rupture: a case report and review of the literature. Eur J Obstet Gynecol Reprod Biol. 2003; 106(1):79-82.

7. Thomsen T, Brown DF, Nadel ES. Abdominal pain in first trimester pregnancy. J Emerg Med. 2003; 24(1):55-58.

8. Lozeau AM, Potter B. Diagnosis and management of ectopic pregnancy. Am Fam Physician. 2005; 72; 1707-1714, 1719-1720.

9. Eyvazzadeh AD, Levine D. Imaging of pelvic pain in the first trimester of pregnancy. Radiol Clin N Am. 2006; 44:863-877.

10. Garcia Oliveira F, Abdelmassih V, Eigenheer AL, et al. Rare association of ovarian implantation site for patients with heterotopic and with primary ectopic pregnancies after ICSI and blastocyst transfer. Hum Reprod. 2001; 16:2227-2229.

11. Tal J, Haddad S, Gordon N: Heterotopic pregnancy after ovulation induction and assisted reproductive technologies: a literature review from 1971 to 1993. Fertil Steril 1996, 66:1-12.

12. Marcus SF, Macnamee M, Brinsden P: Heterotopic pregnancies after in vitro fertilization and embryo transfer. Hum Repro 1995, 10:1232-1236.

13. Jibodu OA, Darne FJ: Spontaneous heterotopic pregnancy presenting with tubal rupture. Hum Rep 1997, 12(5):1098-1099. 
14. Barnhart KT, Katz I, Hummel A, Gracia CR. Presumed Diagnosis of ectopic pregnancy. Obstet Gynecol 2002; 100: 505-10.

15. Perkins JD, Mitchell MR. Heterotopic pregnancy in a large inner-city hospital: a report of two cases. J Natl Med Assoc. 2004; 96:363-366.

16. Scheiber MD, Cedars MI. Successful non-surgical management of a heterotopic an abdominal pregnancy following embryo transfer with cryopreserved-thawed embryos. Hum Reprod. 1999; 14:1375-1377. doi: 10.1093/humrep/14.5.1375.

17. M. J. Govindarajan and R. Rajan, "Heterotopic pregnancy in natural conception," Journal of Human Reproductive Sciences, vol. 1, no. 1, pp. 37-38, 2008.

\section{AUTHORS: \\ 1. Parinita Khot \\ 2. Nitin Kshirsagar \\ 3. Digvijay Kadam \\ 4. Richa Priyamvada}

\section{PARTICULARS OF CONTRIBUTORS:}

1. Resident, Department of Obstetrics and Gynaecology, Krishna Institute of Medical Sciences, Deemed University, Karad.

2. Professor, Department of obstetrics and Gynaecology, Krishna Institute of Medical Sciences, Deemed University, Karad.

3. Senior Resident, Department of obstetrics and Gynaecology, Krishna Institute of Medical Sciences, Deemed University, Karad.

FINANCIAL OR OTHER COMPETING INTERESTS: None
4. Resident, Department of obstetrics and Gynaecology, Krishna Institute of Medical Sciences, Deemed University, Karad.

\section{NAME ADDRESS EMAIL ID OF THE CORRESPONDING AUTHOR:}

Dr. Parnita Khot,

Takahila, 31/B, Flat No.35,

Mahakali Caves Road,

Andheri East,

Mumbai-400093.

E-mail: parinita.khot@gmail.com

Date of Submission: 04/10/2015.

Date of Peer Review: 05/10/2015.

Date of Acceptance: 15/10/2015.

Date of Publishing: 26/10/2015. 